\title{
Equivalence and switching
} between biosimilars and reference molecules in rheumatoid arthritis: protocol for a systematic review and meta-analysis

\author{
Bruna O. Ascef ${ }^{* *}$ D, Matheus O. Almeida², Ana Cristina de Medeiros Ribeiro ${ }^{3}$, Danieli C. O. Andrade ${ }^{3}$, \\ Haliton A. de Oliveira Júnior ${ }^{4}$, Tiago V. Pereira ${ }^{5,6}$ and Patrícia C. de Soárez ${ }^{1}$
}

\begin{abstract}
Background: Biologic drugs such as adalimumab, etanercept, and infliximab represent major first-line and secondline treatments for rheumatoid arthritis (RA) patients. However, their high cost poses a massive burden on healthcare systems worldwide. The expiration of patents for these biologics has driven the production of biosimilar drugs, which are potentially less costly and remarkably similar, albeit not identical to the reference molecules. This paper aims to outline the protocol of a systematic review that will investigate the efficacy and safety profile of biosimilars compared to biologics (objective 1) and the impact of switching between biosimilar drugs and reference biologics on the management of RA patients (objective 2).
\end{abstract}

Methods: We will investigate the effects of any biosimilars of adalimumab, etanercept, and infliximab on RA patients. We will include randomized controlled trials (RCTs) or quasi-RCTs to assess efficacy and safety outcomes and RCTs with two- or multiple-part designs to evaluate the consequences of switching from reference biologics to biosimilar drugs (and vice-versa). Electronic searches will be performed through MEDLINE (via PubMed), EMBASE, LILACS, and CENTRAL (from inception to April 2021). Two independent reviewers will screen studies, extract data, and evaluate the risk of bias. The latter will be carried out considering specific domains from equivalence trials and switching studies. Random-effects models will be fitted to obtain summary estimates using either relative risk or standardized mean difference as a metric. The primary outcome will be the rate of treatment success according to the American College of Rheumatology 20 (ACR20), and the co-primary outcome will be the Health Assessment Questionnaire-Disability Index (HAQ-DI). Conclusions will be based on equivalence hypothesis testing using predefined margins of equivalence elicited from a group of experienced rheumatologists and prior studies. The overall certainty of the evidence will be assessed based on the GRADE system.

Discussion: The present investigation proposes a comprehensive, clinician-oriented approach to assess the equivalence and the impact of switching between biosimilars and biologics on the management of patients with RA. Our results will elucidate the efficacy, safety, immunogenicity of biosimilars, and the clinical consequences of substituting biologics with biosimilars in the management of RA.

\footnotetext{
*Correspondence: brunaascef16@gmail.com

1 Programa de Pós-Graduação em Saúde Coletiva, Departamento de

Medicina Preventiva, Faculdade de Medicina - FMUSP, Universidade de

São Paulo, São Paulo, SP, Brazil

Full list of author information is available at the end of the article
}

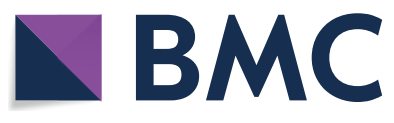

The Author(s) 2021. Open Access This article is licensed under a Creative Commons Attribution 4.0 International License, which permits use, sharing, adaptation, distribution and reproduction in any medium or format, as long as you give appropriate credit to the original author(s) and the source, provide a link to the Creative Commons licence, and indicate if changes were made. The images or other third party material in this article are included in the article's Creative Commons licence, unless indicated otherwise in a credit line to the material. If material is not included in the article's Creative Commons licence and your intended use is not permitted by statutory regulation or exceeds the permitted use, you will need to obtain permission directly from the copyright holder. To view a copy of this licence, visit http://creativecommons.org/licenses/by/4.0/. The Creative Commons Public Domain Dedication waiver (http://creativeco mmons.org/publicdomain/zero/1.0/) applies to the data made available in this article, unless otherwise stated in a credit line to the data. 
Systematic review registration: PROSPERO CRD42019137152 and CRD42019137155

Keywords: Biosimilar pharmaceuticals, Etanercept, Infliximab, Adalimumab, Arthritis, Rheumatoid

\section{Background}

Rheumatoid arthritis (RA) is a chronic inflammatory joint disease that affects up to 20 million people worldwide, thereby representing a major public health burden with important socioeconomic consequences [1-3].

Biological drugs, commonly known as "biologics," are invaluable resources in the treatment of RA patients. Synthetic disease-modifying antirheumatic drugs (DMARDs) are the first line of therapy and associated with biologic DMARDs have changed clinical outcomes, reducing the inflammatory burden of disease and, therefore, chronic articular deterioration [4]. The effectiveness and safety of biologic DMARDs have been robustly established [5-7], and several studies have identified factors that affect the patient's response to these DMARDs [8-11]. However, biologics pose an important challenge for the sustainability of healthcare systems worldwide, given the high direct costs associated with this drug category [1]. For instance, expenses related to biologic treatments can represent almost $40 \%$ of the net drug spending in the USA [12].

Given the rapid evolution of pharmaceutical technologies over the past decade and patent expiration of previously approved biologic molecules, biosimilar drugs have been developed as less costly alternatives to their reference biologics [13]. According to the US Food and Drug Administration (FDA), biosimilars possess clinically similar benefits and safety profile compared to the existing FDA-approved biologics [14]. In this regard, it is believed that biosimilars can accelerate the rheumatic disorder drug market competition, positively impacting the global healthcare system through improved healthcare affordability and increased patients' access to effective and safe drugs $[13,15]$.

However, despite the cost-saving potential of biosimilar drugs, there are still diverging perceptions regarding the efficacy, safety, and immunogenicity of these follow-on biologics [16-18]. Importantly, the switching and interchangeability between biologic and biosimilar drugs are still topics of great debate in the treatment of RA [16-20].

Herein, we describe the protocol of a systematic review that will address the efficacy, safety, and immunogenicity of biosimilars compared to biologics, and the impact of switching between biosimilar drugs and reference biologics on the management of RA patients. Unlike previous reviews, we will establish acceptable equivalence margins elicited from clinical specialists to conclude on the equivalence of biosimilars compared to biologics.

\section{Methods/design}

\section{Reporting guidelines used in this protocol}

The present protocol followed the Preferred Reporting Items for Systematic Reviews and Meta-Analyses Protocols guidelines (PRISMA-P) 2015 statement (Additional file 1) [21]. We will refer separately to the main objectives of the systematic review as efficacy and safety (objective 1 ) and switching (objective 2) because they need varying methodologies and approaches.

\section{PROSPERO synopses}

Synopses for the two main objectives were prospectively and separately registered in the International Prospective Register of Systematic Reviews (https://www.crd.york. ac.uk/PROSPERO/). The first objective will focus on the efficacy and safety of biosimilars compared to biologics (PROSPERO number: CRD42019137152), whereas the second objective will examine the clinical impact of switching from reference biologics to biosimilars on the management of RA patients whose treatment has already been started (PROSPERO: CRD42019137155).

\section{Adopted reporting and developing standards}

The proposed systematic review will be reported following Preferred Reporting Items for Systematic Review and Meta-analysis (PRISMA Statement) [22]. We will also follow the guidelines of the Cochrane Handbook for conducting systematic reviews of interventions [23]. Besides, since there are specific aspects related to the conduct, interpretation, and reporting of equivalence and noninferiority trials, we will also adopt the US Agency for Healthcare Research and Quality recommendations [24].

\section{Electronic searches}

Search strategies were built using controlled vocabulary according to each database and free-text terms based on the research question. We will use the following electronic databases (from inception to April 2021): MEDLINE via PubMed, EMBASE, Cochrane Central Register of Controlled Trials (CENTRAL), and Latin American and Caribbean Health Science (LILACS). A detailed description of the search strategy is available in Additional file 2 .

\section{Other sources}

We will also search for non-published or ongoing trials in the EU Clinical Trial Register (https://www.clinicaltr ialsregister.eu), International Clinical Trials Registry 
Platform-World Health Organization (http://apps.who. int/trialsearch/), and Clinicaltrials (https://clinicaltrials. gov/). The search strategies to be used in these platforms are described in Additional file 2. When necessary, we will contact corresponding authors for supplementary information. Additionally, we will manually screen the references of all included trials as well as previous systematic reviews. Finally, we will employ Google Scholar and Epistemonikos (https://www.epistemonikos.org/) to retrieve relevant reports citing all relevant included articles. No language limitation will be imposed.

\section{Eligibility criteria \\ Types of biosimilars}

We will assess any biosimilars of adalimumab, etanercept, and infliximab. We chose these three main biologics because they are the most prescribed first-line biologic DMARDs in RA [25]. Also, these three DMARDS have the highest numbers of approved biosimilars for RA in the market $[13,25]$.

\section{Types of control interventions}

We will consider as control interventions the reference biologic drugs (i.e., adalimumab, etanercept, and infliximab originals). No restrictions on dosages, treatment schedules, co-treatment, or combined therapies will be imposed.

\section{Types of trials}

Types of trials: objective 1 (efficacy and safety) To assess the efficacy and safety of biosimilars ("biosimilarity") [27], we will include randomized controlled trials (RCTs) or quasi-RCTs. We will include all trials comparing biosimilars to biologic drugs irrespective of the type of statistical design (superiority, equivalence, or non-inferiority). A quasi-randomized trial was defined as a prospective interventional study whose allocation sequence was not truly random (e.g., consecutive order, day of the week, date of birth, etc.). For trials with a 2-part study design, we will consider results from the first period (biosimilarity) only to avoid carry-over effects.

Types of trials: objective 2 (switching) To assess the impact of switching on clinical outcomes of RA patients, we will include RCTs with two- or multiple-part designs. The following four main designs of switching trials will be considered:

- Single-switch design $[28,29]$ : Trials in which there is a single switch from each treatment to the other. All patients receive the study interventions in succes- sive periods. Firstly, patients are randomly allocated to either a biosimilar or a biologic drug (first period). Then, in the second period, treatments are randomly switched in both directions (group 1: biologic $\rightarrow$ biosimilar; group 2: biosimilar $\rightarrow$ biologic OR group 1: biologic $\rightarrow$ biosimilar, group 2: biosimilar $\rightarrow$ biologic; group 3: biologic $\rightarrow$ biologic; group 4: biosimilar $\rightarrow$ biosimilar).

- Transition design 1 (two non-switching groups as a control): Trials in which there is a single switch from one treatment (biologic drug) to another (biosimilar drug), but not the contrary. Firstly, patients are randomly allocated to either a biosimilar or a biologic drug (first period). Then, in the second period, the trial becomes a three-arm trial in which patients in the biologic drug group are re-randomized either to continue in the biologic group or to switch to the biosimilar drug treatment. Patients initially allocated to the biosimilar group continue to receive a biosimilar throughout the study period (experimental group: biologic $\rightarrow$ biosimilar; control arm 1: biologic $\rightarrow$ biologic; control arm 2: biosimilar $\rightarrow$ biosimilar).

- Transition design 2 (randomized trials with an openlabel extension; single non-switching group as a control): Trials in which there is a single switch from a biologic drug to a biosimilar drug, but not the contrary. Firstly, patients are randomly allocated to either a biosimilar or a biologic drug (first period). Then, in open-label extended phase (second period), all patients (intervention and control groups) receive the biosimilar drug (experimental group: biologic $\rightarrow$ biosimilar; control arm 1: biosimilar $\rightarrow$ biosimilar).

- Multiple switches design: Also known as interchangeability design [28, 29], in which multiple switches between treatments are allowed throughout the trial follow-up.

\section{Type of participants}

Trials will be included if patients with RA had been diagnosed with validated and established international criteria. No limitation will be imposed on age, baseline RA severity, sex, lines of treatment (e.g., treatment-naïve patients or second line of treatment), or any other major demographic characteristics.

\section{Types of outcome measures}

All outcomes were prespecified in the registered PROSPERO synopses and were categorized into three types: efficacy (encompassing outcomes related to disease activity, functional capacity, quality of life, and structural damage progression), safety, and immunogenicity. For efficacy outcomes, we will extract data 
at the following time points: 1 month ( \pm 2 weeks), 3 months ( \pm 4 weeks), 6 months ( \pm 4 weeks), 8 months ( \pm 4 weeks), 12 months ( \pm 4 weeks), 36 months ( \pm 4 weeks), and 48 months ( \pm 4 weeks). For safety and immunogenicity outcomes, we will collect data from longest follow-up available.

Primary outcomes We prespecified a primary outcome, a co-primary outcome, and all secondary outcomes. A co-primary outcome was adopted because the demonstration of superiority or equivalence in a single outcome is insufficient to support clinical decisions for the management of RA patients. The choice of primary and coprimary outcomes was decided on a panel composed of two RA specialists supervised by two researchers with experience in evidence synthesis. The rationale was to evaluate the equivalence between biosimilars and reference biologic drugs using the minimum set of clinical outcomes that incorporate both physician-reported and patient-reported outcomes. Similar approaches have been used previously in RA trials [30,31].

Objective 1: efficacy and safety The primary outcome will be treatment success at 6 months according to the American College of Rheumatology 20 (ACR20) [32].

The co-primary outcome will be HAQ-DI, which assesses the functional status of patients through the evaluation of eight domains of daily life activities. The highest score reported for any component question in each domain determines the final score for that domain. By convention, the overall disability index is expressed on a 0 to 3 scale, representing an average score across the domains. A HAQ-DI of 0 indicates no functional disability, whereas a HAQ-DI of 3 denotes severe functional disability [33].

If trials report results at different time points, we will use the time point closest to 6 months.

Objective 2: the impact of switching The primary outcome will be the rate of treatment success at 6 months after the first switch (i.e., 6 months after re-randomization or 6 months after the first switch on the open-label extension phase) defined by the ACR20 (dichotomous outcome). The co-primary outcome will be the HAQ-DI index also measured at 6 months after the first switch (continuous outcome). If outcome data are reported at different time points, we will use the time point closest to 6 months.

Secondary outcomes (efficacy, safety, and immunogenicity) Secondary outcomes: efficacy
Secondary outcomes of efficacy will be examined at 6 months of follow-up (or the time point closest to 6 months) and will include disease activity, prevention of structural damage progression, and quality of life measures:

- Measures of disease activity: the American College of Rheumatology criteria with 50\% (ACR50) and 70\% (ACR70) responses, simplified disease activity score (SDAI), clinical disease activity score (CDAI), disease activity score in 28 joints based on the erythrocyte sedimentation rate (DAS28-ESR), disease activity score in 28 joints with four components based on C-reactive protein (DAS28-CRP), and the numeric index of the ACR response (ACR-N).

- Functional capacity/quality of life: scores of HAQDI and the Medical Outcomes Study 36-item ShortForm Health Survey (SF-36) (physical and mental components summaries).

- Prevention of structural damage progression: scores of Sharp/Van der Heijde or Sharp-Van Der Heidje Modified Score Method (mTRSS). A full description of secondary outcomes can be found in Additional file 3.

\section{Secondary outcomes: safety}

We will evaluate the safety of biosimilars compared to biologics by the proportion of patients with treatmentemergent adverse events (TEAEs), serious TEAEs, infusion-related reactions (IRRs), injection site reactions (ISRs), hypersensitivity, malignancies, active tuberculosis, serious infections, all-cause mortality, and treatmentrelated mortality. Also, we will evaluate discontinuation rates in both treatments. A full list of safety outcomes can be found in Additional file 3.

\section{Secondary outcomes: immunogenicity}

Immunogenicity will be evaluated by the proportion of patients with positive anti-drug antibodies (ADAs) and the proportion of patients with positive neutralizing antibodies (Nabs).

\section{Investigator training and data calibration}

All investigators involved in the study selection, data extraction, and risk of bias assessments will be trained. Specifically, we will include a sample of four trials and perform calibration among reviewers, followed by oral and written instructions. We will perform multiple rounds of "calibration checks" throughout the data 
extraction process. The data extraction process will be guided by a codebook, which contains detailed technical information on each variable, definitions, assumptions, and possible responses (in case of categorical responses).

\section{Study screening and selection}

We developed a customized web platform for data extraction and curation using Ragic (www.ragic.com). This database was carefully designed to simultaneously allow for study screening and selection and data extraction for the systematic review. Two independent investigators will perform all steps. Specifically, during the screening phase, two review authors will independently evaluate titles and abstracts. Disagreements will be solved by a consensus. Next, for each study selected, fulllength articles will be downloaded, and two independent reviewers will re-assess the eligibility of each pre-selected trial. In cases of disagreements, a third reviewer will be consulted. Reasons for exclusions will be described in detail in subsequent publications.

\section{Data extraction and management Analysis population}

Trials may report two populations for the analysis: an intention-to-treat (ITT) population and a per-protocol (PP) population [24]. For the analysis of both objectives 1 and 2, preference will be given to results based on the PP population, because of the conservative effect of the per-protocol approach on equivalence testing [24]. Since there may be a substantial variety in the definition of what constitutes a PP population or an ITT analysis, we will collect and tabulate in detail the definition of PP and ITT used in each trial.

\section{Numerical and graphical results}

All data will be extracted independently by two investigators. Discrepancies will be solved via a consensus. We will extract all pertinent quantitative information, including the number of participants at baseline, the number of participants analyzed, and measures of central tendency, variability, and precision. Specifically, whenever available, we will collect means, mean changes, the difference between means at follow-up, medians, standard deviations, interquartile ranges, standard errors, confidence intervals (and their coverage, e.g., 90 or $95 \%$ ), $P$-values (one- or two-sides), and $t$ statistics. These data will be used to approximate means and standard deviations when necessary [34]. For continuous outcomes, we will use follow-up data preferentially but will use the mean change from baseline when follow-up values are not available [35].

Quantitative data from figures and graphs will be extracted independently using digitizing software
(Digitizelt 2.2.2, Germany, https://www.digitizeit.de/). Estimates from the digitizing software will be averaged out to generate the final value. When necessary, data for the same trial will be extracted from multiple sources (e.g., multiple related publications and trial registries). Multiple publications from the same trial will be linked via a unique identifier. The linkage of several articles to the same identifier will be performed via the number assigned to the study's registration (e.g., The National Clinical Trial number [NCT] from clinicaltrials.gov). When the trial registration number was not explicitly reported, we will perform a careful evaluation of the similarity between the eligible trial and those already included in the systematic review. The similarity will be rated by two reviewers based on the patient enrollment period, the affiliation of the trial investigators, participant institutions, target sample size, funding source, and patients' baseline characteristics. In case of multiple publications reporting results for the same time point, we will extract data from the most complete or most recent article.

\section{Ongoing trials}

We will summarize all identified ongoing trials, detailing the primary author, research question(s), methods, outcome measures, and study start date, along with an estimate of the study completion date.

\section{Assessment of risk of bias}

Two review authors will independently assess the risk of bias in the included studies. Each domain will be classified as being at a low, unclear, or high risk of bias. Disagreements will be resolved by consensus or discussion with a third reviewer. The studies will be assessed by outcome level. If the trial has one or more domains with a high risk of bias, it will be considered as a high risk of bias study. If the trial has more than two domains at uncertain risk of bias, we will judge the risk of bias to be uncertain. If the trial has a low risk of bias in all domains or one domain as uncertain bias, it will be considered as a low risk of bias study.

\section{Assessment of risk of bias in efficacy and safety trials}

We will use criteria recommended by the Cochrane collaboration (Cochrane Risk of bias tool 1.0) [36]. The following domains will be evaluated: random sequence generation, allocation concealment, blinding of participants and investigators, blinding of outcome assessors, and incomplete outcome data (PP and ITT population analysis). To specifically address equivalence or noninferiority trials, we will refer to the recommendations by the US Agency for Healthcare Research and Quality [24] (Table 1). Specifically, we will assess the inconsistent 
Table 1 Risk of bias domains to be evaluated on equivalence or non-inferiority trials

\begin{tabular}{lll}
\hline Type of bias & Domain & Source \\
\hline Selection bias & Random sequence generation & Cochrane RoB tool 1.0 \\
& Allocation concealment & Cochrane RoB tool 1.0 \\
Inconsistent application of inclusion/exclusion criteria & US Agency for Healthcare Research and Quality \\
Performance bias & Blinding of participants and investigators & Cochrane RoB tool 1.0 \\
Detection bias & Participants behavior changes & US Agency for Healthcare Research and Quality \\
& Blinding of outcome assessment & Cochrane RoB tool 1.0 \\
Attrition bias & Outcome measurement techniques & US Agency for Healthcare Research and Quality \\
& Incomplete outcome data & Cochrane RoB tool 1.0 and US Agency for \\
\hline
\end{tabular}

Sources: Cochrane Risk of Bias in randomized trials (RoB 1.0.) described in the Cochrane Handbook for Systematic Reviews of Interventions [36] and the US Agency for

Healthcare Research and Quality recommendations [24]

application of inclusion/exclusion criteria, patients selected for anticipated nonresponse or good response in one arm, patient behavior changes (poor adherence, use of concomitant treatments, and protocol violations), inadequate outcome measurement techniques, and incomplete outcome data (PP and ITT population analysis: ITT population analysis may underestimate the treatment effect in equivalence/non-inferiority trials). More information on the criteria used in each domain can be found in Additional file 4.

\section{Assessment of risk of bias in switching trials}

For switching trials, we will use the recommendations of Moots et al. [28] and the FDA guidance for considerations in demonstrating interchangeability with a reference product [14]. The six specific domains to be evaluated are:

- Randomized and blinded design with appropriate control arms;

- At least 1-way switch from originator to biosimilar;

- The assessment of immunogenicity;

- The wash-out period between treatment;

- Enough power to assess efficacy and safety (equivalence phase); and

- Enough follow-up periods.

More information about the criteria of judgments can be seen in Additional file 5 .

\section{Data synthesis}

\section{Effect size measures}

For binary outcomes, we will combine study estimates using the relative risk (RR) as a measure of effect. For continuous outcomes, we will use the standardized mean difference (SMD) defined as the bias-adjusted method of Hedges. SMD will be used because it has similar statistical power and is more generalizable than the mean difference [37].

\section{Meta-analysis models}

Main analyses will be based on the random-effects model with the restricted maximum-likelihood estimator for the between-study variance [38]. A randomeffects model was prespecified as the primary model of analysis since we anticipated variability in the design and population characteristics of the included trials. Results for a fixed-effects model (inverse-variance method) will be presented simultaneously as a sensitivity analysis.

\section{Statistical heterogeneity}

We will test for the presence of statistical heterogeneity across trial estimates using Cochran's Q test [39] and the magnitude of the between-trial heterogeneity will be quantified with the $\mathrm{I}^{2}$ metric [40]. When feasible (i.e., 10 or more trials), we will investigate potential sources of statistical heterogeneity with the random-effects metaregression analysis and subgroups analysis. Explanatory variables to be included in meta-regression models are described below.

\section{Small-study and publication biases}

We will investigate the association between trial size (precision) and treatment effects in contour-enhanced funnel plots, contrasting the effect estimates on the horizontal axis against their standard errors on the vertical axis, accompanied by a regression test for asymmetry. Furthermore, for continuous outcomes, small-study biases will be investigated by Egger's regression test, whereas for binary outcomes we will use Harbord's test [41]. 


\section{Equivalence testing}

\section{Criteria to claim equivalence}

Equivalence will be evaluated and interpreted using predefined margins of equivalence (Fig. 1). Upper and lower equivalence bounds were specified based on the smallest effect size of clinical importance. These values were computed from large placebo-controlled trials and validated by two rheumatologists with extensive experience treating patients with RA. Prespecified boundaries of equivalence will be applied to the primary and co-primary outcomes only.
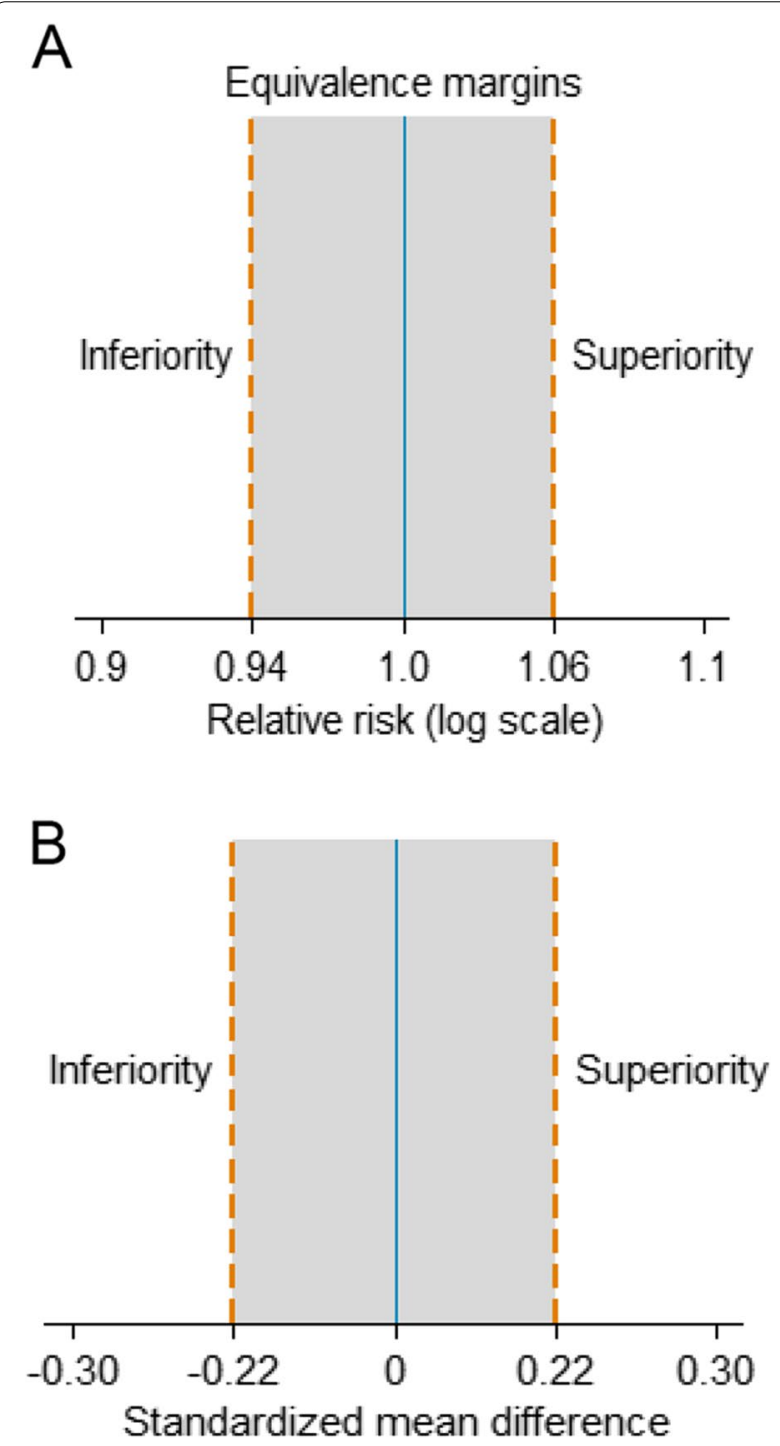

Fig. 1 Boundaries of equivalence (dashed lines) for a two-sided 95\% confidence interval of the treatment difference. A Equivalence margins for ACR20 (primary outcome). B Equivalence margins for HAQ-DI (co-primary outcome). If the summary 95\% Cl lies within the gray regions, the null hypothesis will be rejected, and equivalence will be claimed
Based on random-effects models, lower and upper confidence limits will be calculated. For a specific outcome (i.e., either ACR20 or HAQ-DI), if the two-sided 95\% confidence interval for the difference in effect is completely contained within the prespecified boundaries of equivalence, biosimilars and biologics will be considered equivalent. However, the rejection of non-equivalence (for both ACR20 and HAQ-DI outcomes) will be required for biosimilars to be declared overall equivalent to biologic drugs. Secondary outcomes will be examined through standard superiority tests (two-tailed).

\section{Margins of equivalence: $A C R 20$ criteria}

For the ACR20 outcome, we prespecified equivalence margins to preserve $90 \%$ of the effects observed with biologics using the fixed-margin method [42]. Specifically, we calculated the equivalence margins as:

$$
\begin{aligned}
& \text { Equivalence margins }=e^{ \pm m} \\
& \text { with } \\
& m=\log \left(R R^{P E-1}\right)
\end{aligned}
$$

where PE stands for the preserved effect (range 0 to $1[100 \%])$. Based on a network meta-analysis by Guyott et al. [43] that included 11 randomized trials with 3762 patients who were unresponsive to methotrexate, the RR under a random-effects model for ACR20 at 6 months for any biologics (adalimumab/etanercept/infliximab) vs. placebo was approximately 1.80. Similar estimates were obtained considering a combination of both methotrexate-naive and methotrexate unresponsive patients, in which the frequentist summary RR of achieving ACR20 was 1.81 (random-effects model, 13 trials, 7087 patients) for the comparison adalimumab/etanercept/infliximab vs. placebo [44]. Thus, biosimilars will be considered equivalent to biologics if the $95 \%$ confidence limits for the summary RR lie within the 0.94 and 1.06 interval (Fig. 1A).

\section{Margins of equivalence: $H A Q-D I$}

For HAQ-DI, which is a continuous outcome (with higher scores meaning worse function status), equivalence margins were constructed under the clinical assumption that an increase equal or larger than 0.15 units over 1 year on the 0-to-3 HAQ-DI scale is considered clinically perceptible by the patients [45]. Based on trials encompassing a diversified group of RA patients that received adalimumab, etanercept, or infliximab (1816 patients) [46-48], we estimated the HAQ-DI population standard deviation after a 24/26-week treatment to be approximately 0.69 . Therefore, on a standardized 
mean difference scale, the 0.15 units difference is approximately equivalent to \pm 0.22 standard deviations (Fig. 1B).

\section{Analysis of subgroups and meta-regression}

We will perform prespecified subgroup analyses. When feasible, the following subgroup analysis will be conducted:

- Type of molecule (infliximab vs. etanercept vs. adalimumab);

- Concomitant use of synthetic disease-modifying antirheumatic drugs (yes or no);

- Sample size (average of $>100$ patients per group vs. $<100$ patients per group);

- Allocation concealment (low risk vs. high risk/ unclear risk);

- Trial duration (equivalence studies) (3 vs. 6 vs. 12 months);

- Type of design (multiple switching/switching studies vs. transitional studies)

- Funding independent of industry (yes vs. no/ unclear); and

- Publication status (published vs. unpublished).

The above-mentioned variables will also serve as explanatory variables in meta-regression models.

\section{Sensitivity analysis (primary and co-primary outcomes) Non-inferiority}

As there would be minor concerns if biosimilar drugs were more efficacious than conventional biologicals, exploratory non-inferiority analysis will be conducted in the event the data do not support the equivalence. Specifically, we will claim non-inferiority if the lower limit of the $95 \% \mathrm{CI}$ is above the prespecified cutoffs for both ACR20 and HAQ-DI.

\section{Time points}

Sensitivity analyses for the primary and co-primary outcomes will also be performed at different time points: $1,3,8,12,36$, and 48 months. All these analyses will be considered exploratory and will be conducted from the superiority testing point of view.

\section{Assessment of overall certainty of evidence}

The overall certainty of evidence will be assessed by two investigators and will be based on the Grading of Recommendations Assessment, Development and Evaluation (GRADE) system [49]. Assessments will be conducted by outcome. Disagreements will be settled by consensus or discussion with a third reviewer. The certainty of evidence of each outcome will be graded as very low, low, moderate, or high. The following domains will be assessed:

- Study design and risk of bias

- Inconsistency

- Indirectness

- Imprecision

- Other factors (e.g., reporting bias, publication bias)

\section{Ranking of outcomes by their relative importance}

We have adopted the recommendations from the GRADE handbook for selecting and rating the importance of outcomes [27]. Specifically, we ranked each outcome as "critical," "important but not critical," and "limited importance to decision-making" (Table 2).

The ranking was conducted through consultations with two clinical specialists (rheumatologists) and a physical therapist specialized in evidence synthesis. These professionals were invited to participate based on their clinical experience, academic background, and the lack of any conflict of interest. Briefly, before the scoping meeting, based on previous systematic reviews, we screened the list of outcomes (both primary and secondary outcomes) of 14 trials that we knew a priori that met all the eligibility criteria. Subsequently, we created an integrated list of all outcomes and categorized them into five main domains:

- Disease activity/clinical response

- Function capacity/quality of life

- Structural damage progression

- Immunogenicity

- Safety

Through an iterative approach in a single scoping meeting, each member of the collaborative working group ranked outcomes independently. Conflicting ranking cases were discussed jointly until a consensus was reached.

\section{Discussion}

At the time of writing this manuscript, more than 16 biosimilars of adalimumab, etanercept, and infliximab had been approved in the USA, Europe, Canada, and Latin America for the treatment of RA [50-53]. The proposed systematic review will comprehensively assess the efficacy, safety, and immunogenicity of these biosimilars compared to their originator molecules and examine the clinical consequences of switching from biologics to biosimilars in the management of RA patients. 
Table 2 Ranking of outcomes

\begin{tabular}{|c|c|c|}
\hline Outcome & $\begin{array}{l}\text { Rating scale } 1-9^{a} \text { (importance of the outcome for } \\
\text { decision-making) }\end{array}$ & Type of outcome \\
\hline \multicolumn{3}{|l|}{ Disease activity/clinical response } \\
\hline CDAI & 9 (critical) & Continuous or binary \\
\hline DAS-28 ESR & 8.5 (critical) & Continuous or binary \\
\hline SDAI & 8.5 (critical) & Continuous or binary \\
\hline ACR 20 & 8 (critical) & Binary \\
\hline ACR 50 & 8 (critical) & Binary \\
\hline DAS-28 CRP & 8 (critical) & Continuous or binary \\
\hline ACR-N & 7.5 (critical) & Continuous \\
\hline ACR 70 & 6 (important but not critical) & Binary \\
\hline EULAR response & 5 (important but not critical) & Binary \\
\hline \multicolumn{3}{|l|}{ Safety } \\
\hline TEAE & 9 (critical) & Binary \\
\hline Serious TEAE & 9 (critical) & Binary \\
\hline Death related to treatment & 9 (critical) & Binary \\
\hline IRRs & 9 (critical) & Binary \\
\hline Hypersensitivity & 9 (critical) & Binary \\
\hline Active tuberculosis & 9 (critical) & Binary \\
\hline Serious infections & 9 (critical) & Binary \\
\hline Death all causes & 8 (critical) & Binary \\
\hline Overall discontinuation rate & 8.5 (critical) & Binary \\
\hline Malignancies & 8 (critical) & Binary \\
\hline Fatigue & 7 (critical) & Binary \\
\hline \multicolumn{3}{|l|}{ Function capacity/quality of life } \\
\hline HAQ-DI & 8.5 (critical) & Continuous \\
\hline SF-36 score_-physical component summary & 8 (critical) & Continuous \\
\hline SF-36 score- mental component summary & 8 (critical) & Continuous \\
\hline \multicolumn{3}{|l|}{ Structural damage progression } \\
\hline mTRSS or Sharp/van der Heijde score & 5.5 (important but not critical) & Continuous \\
\hline \multicolumn{3}{|l|}{ Immunogenicity } \\
\hline Incidence of ADAs & 5 (important but not critical) & Binary \\
\hline NABs_-positive & 5 (important but not critical) & Binary \\
\hline
\end{tabular}

$A C R$ The American College of Rheumatology, CRP C-reactive protein level, HAQ-DI Health Assessment Questionnaire-Disability Index, VAS Visual analog scale, SDAI Simplified Disease Activity Score, DA disease activity, CDAI Clinical Disease Activity Score, DAS28-ESR Disease Activity Score in 28 joints based on the erythrocyte sedimentation rate, DAS28-CRP Disease Activity Score in 28 joints, four components based on C-reactive protein, $A C R-N T$ The numeric index of the ACR response, EULAR European League Against Rheumatism, SF-36 The Medical Outcomes Study 36-item Short-Form Health Survey, mTRSS Sharp/van der Heijde score, IRRs Infusion-related reactions, TEAE Overall treatment emergent adverse event

${ }^{\text {a }}$ Based on the mean average ranking of two rheumatologists with extensive clinical experience in treating RA patients and a physical therapist with advanced training in evidence synthesis

The systematic review described here, to the best of our knowledge, is the first one proposing a comprehensive, clinician-oriented approach to assess the equivalence and the impact of switching between biosimilars and biologics on the management of patients with RA. Over the past years, relevant systematic reviews on the differences between biosimilar drugs vs. reference molecules in rheumatoid arthritis have been published [54-57]. However, only a few of the previous systematic reviews considered all pertinent domains of risk of bias that are specific to biosimilar drugs [57]. Similarly, only a few reviews graded the certainty of evidence according to the GRADE system [57]. Besides, we propose the first systematic evaluations based on equivalence testing at the level of meta-analysis using equivalence margins elicited from clinical specialists. We believe that our systematic review will add to the body of evidence in biosimilar drugs, building confidence for patients and clinicians, and providing healthcare systems with 
updated information that can help in their decisionmaking processes.

Furthermore, by examining the randomized evidence on the effects of a wide range of biosimilars, we will also be able to address whether switching from reference biologics to biosimilars or vice and versa, in general, results in similar clinical benefits with acceptable immunogenicity and safety profiles. As a result, our findings hold great potential to affect not only the therapeutic regimen of RA patients that will use a DMARD for the first time but also the treatment of those that will have their treatment substituted from a biologic to a biosimilar, or from a biosimilar to a biologic.

Overall, we expect that our results will guide clinicians, researchers, decision-makers, stakeholders, and policymakers about the efficacy, safety, immunogenicity, and substitution and interchangeability of currently marketed biosimilars for the treatment of RA patients and assist healthcare systems to employ more efficiently the scarce existing resources.

\begin{abstract}
Abbreviations
RA: Rheumatoid arthritis; DMARDs: Disease-modifying antirheumatic drugs; FDA: The US Food and Drug Administration; PRISMA-P: The Preferred Reporting Items for Systematic Reviews and Meta-Analyses Protocols guidelines; PROSPERO: The International Prospective Register of Systematic Reviews; PRISMA: Preferred Reporting Items for Systematic Review and Meta-analysis; US: United States; EU: European; CENTRAL: Cochrane Central Register of Controlled Trials; LILACS: Latin American and Caribbean Health Science; RCTs: Randomized controlled trials; ACR20: The American College of Rheumatology 20 criteria; ACR 50: The American College of Rheumatology 50 criteria; ACR70: The American College of Rheumatology 70 criteria; VAS: Visual analog scale; HAQ-DI: Health Assessment Questionnaire—Disability Index; DAS28-ESR: Disease activity score in 28 joints based on the erythrocyte sedimentation rate; DAS28-CRP: Disease activity score in 28 joints with four components based on C-reactive protein; ACR-N: The numeric index of the ACR response; SF-36: The Medical Outcomes Study 36-item Short-Form Health Survey; mTRSS: Sharp-Van Der Heidje Modified Score Method; TEAEs: Treatment-emergent adverse events; IRRs: Infusion-related reactions; ADAs: Anti-drug antibodies; Nabs: Neutralizing antibodies; PP: Per-protocol; ITT: Intention-to-treat; RR: Relative risk; SMD: The standardized mean difference; PE: Preserve effect; Log: Logarithm; GRADE: The Grading of Recommendations Assessment, Development and Evaluation System.
\end{abstract}

\section{Supplementary Information}

The online version contains supplementary material available at https://doi. org/10.1186/s13643-021-01754-x.

Additional file 1. PRISMA for systematic review protocols (PRISMA-P) Checklist. Source: Moher et al. [21].

Additional file 2. Search Strategies of electronic database and other sources.

Additional file 3. Secondary outcomes of efficacy (disease activity measures, functional capacity, quality of life and structural damage progression) and safety. ACR: the American College of Rheumatology; CRP: C-Reactive Protein level; VAS: visual analog scale; HAQ-DI: Health Assessment Questionnaire - Disability Index; VAS: visual analog scale; SDAl: Simplified Disease Activity Score DA: disease activity; CDAl: Clinical
Disease Activity Score DAS28-ESR: Disease Activity Score in 28 joints based on the erythrocyte sedimentation rate; DAS28-CRP: Disease Activity Score in 28 joints, four components based on C-reactive protein; SJC: Swollen joint count; TJC: Tender Joint Count; ACR-N: The numeric index of the ACR response; EULAR: European League Against Rheumatism; SF-36: The Medical Outcomes Study 36-item Short-Form Health Survey; mTRSS: Sharp/ van der Heijde score; TEAE: Overall Treatment Emergent Adverse Event; infusion-related reactions.

Additional file 4. Criteria to identify bias on equivalence or non-inferiority studies. Sources: Cochrane Risk of Bias in randomized trials (ROB 1.0.) [36] and the US Agency for Healthcare Research and Quality recommendations [24].

Additional file 5. Criteria to identify bias on switching studies. Notes: * It must be clearly pointed out. The wash-out period is defined as the time between the discontinuation of one biologic and the initiation of a second biologic. This wash-out period is arbitrarily based on the half-life of the biologic, namely the time needed to eliminate $50 \%$ of the biologic from the bloodstream.; ** The comparative assessment should occur during the final exposure period after enough time (i.e., an adequate washout period of at least three or more half-lives) has elapsed following the last administration of the reference product in the switching arm; The number of doses of the proposed interchangeable product or reference product administered in the final exposure period will depend on the half-life and clinical dosing regimen.; The serum half-time of infliximab is around 14 days or 2 weeks; Etanercept has a mean \pm standard deviation half-life of $102 \pm 30$ hours was observed ( more or less 4 days); The mean terminal half-life of adalimumab was approximately 2 weeks. Sources: Moots et al. [28] and FDA [14].

\section{Authors' contributions}

BOA, TVP, and PCS conceived and designed the study. BOA and MOA created the electronic search strategies. BOA and MOA will conduct the study screening and selection, extraction data, assessment of bias, and overall quality of evidence. In case of disagreements, TVP will be the third reviewer. BOA, MOA, and TVP customized the web platform for data extraction. ACMR, DCOA, and MOA composed the panel of experts for selecting and rating the importance of outcomes. HAOJ provided methodological advice on rheumatoid arthritis and made important intellectual contributions for the manuscript. BOA, TVP, and PCS drafted the manuscript, which was edited and revised by all authors. TVP and PCS provided supervision. All authors read and approved the final version of the manuscript.

\section{Funding}

TVP is funded by the Chevening Scholarship Program (Foreign and Commonwealth Office, UK). This project had no other funding to declare.

\section{Declarations}

Ethics approval and consent to participate

Not applicable.

\section{Consent for publication}

Not applicable.

\section{Competing interests}

The authors declare that they have no competing interests.

\section{Author details}

1Programa de Pós-Graduação em Saúde Coletiva, Departamento de Medicina Preventiva, Faculdade de Medicina - FMUSP, Universidade de São Paulo, São Paulo, SP, Brazil. ${ }^{2}$ Programa de Pós-Graduação em Fisioterapia, Universidade Ibirapuera, São Paulo, SP, Brazil. ${ }^{3}$ Disciplina de Reumatologia do Hospital das Clínicas da Faculdade de Medicina, Universidade de São Paulo, São Paulo, SP, Brazil. ${ }^{4}$ Programa de Pós-Graduação em Medicamentos e Assistência Farmacêutica, Departamento de Farmácia Social, Universidade Federal de Minas Gerais, Belo Horizonte, MG, Brazil. ${ }^{5}$ Applied Health Research Centre, Li Ka Shing Knowledge Institute, St Michael's Hospital, Toronto, ON, Canada. ${ }^{6}$ Department of Health Sciences, College of Medicine, University of Leicester, Leicester, UK. 
Received: 17 September 2020 Accepted: 27 June 2021

Published online: 17 July 2021

\section{References}

1. Aletaha D, Neogi T, Silman AJ, Funovits J, Felson DT, Bingham CO III, et al. 2010 rheumatoid arthritis classification criteria: an American College of Rheumatology/European League Against Rheumatism collaborative initiative. Arthritis Rheum. 2010;62(9):2569-81.

2. Gabriel SE. The epidemiology of rheumatoid arthritis. Rheum Dis Clin North Am. 2001;27:269-82.

3. Safiri S, Kolahi AA, Hoy D, Smith E, Bettampadi D, Mansournia MA, et al. Global, regional and national burden of rheumatoid arthritis 1990-2017: a systematic analysis of the Global Burden of Disease study 2017. Ann Rheum Dis. 2019;78(11):1463-71.

4. Smolen JS, Landewé R, Bijlsma J, Burmester G, Chatzidionysiou K, Dougados $M$, et al. EULAR recommendations for the management of rheumatoid arthritis with synthetic and biological diseasemodifying antirheumatic drugs: 2016 update. Ann Rheum Dis. 2017;76(6):960-77.

5. Singh JA, Saag KG, Bridges SL Jr, AkI EA, Bannuru RR, Sullivan MC, et al. 2015 American College of Rheumatology guideline for the treatment of rheumatoid arthritis. Arthritis Rheumatol. 2016;68(1):1-26

6. Singh JA, Hossain A, Ghogomu ET, Mudano AS, Tugwell P, Wells GA. Biologic or tofacitinib monotherapy for rheumatoid arthritis in people with traditional disease-modifying anti-rheumatic drug (DMARD) failure: a Cochrane systematic review and network meta-analysis (NMA). Cochrane Database Syst Rev. 2016;11(11):CD012437.

7. Nam JL, Takase-Minegishi K, Ramiro S, Chatzidionysiou K, Smolen JS, Van Der Heijde D, et al. Efficacy of biological disease-modifying antirheumatic drugs: a systematic literature review informing the 2016 update of the EULAR recommendations for the management of rheumatoid arthritis. Ann Rheum Dis. 2017;76(6):1113-36.

8. Daien $\mathrm{Cl}$, Morel J. Predictive factors of response to biological disease modifying antirheumatic drugs: towards personalized medicine. Mediators Inflamm. 2014;(2014):1-11.

9. Souto A, Maneiro JR, Gomez-Reino JJ. Rate of discontinuation and drug survival of biologic therapies in rheumatoid arthritis: a systematic review and meta-analysis of drug registries and health care databases. Rheumatology. 2016;55(3):523-34.

10. Ramiro S, Gaujoux-Viala C, Nam JLSJ, Buch M, Gossec L, Van Der Heijde D, et al. Safety of synthetic and biological DMARDs: a systematic literature review informing the 2013 update of the EULAR recommendations for management of rheumatoid arthritis. Ann Rheum Dis. 2014;73(3):529-35

11. Hamann P, Holland R, Hyrich K, Pauling JD, Shaddick G, Nightingale $A$, et al. Factors associated with sustained remission in rheumatoid arthritis in patients treated with anti-tumor necrosis factor. Arthritis Care Res. 2017;69(6):783-93.

12. Stiff KM, Cline A, Feldman SR. Tracking the price of existing biologics when drugs enter the market. Expert Rev Pharmacoecon Outcomes Res. 2019;19(4):375-7.

13. Allocati E, Gerardi C, Garattini S, Banzi R. Clinical evidence supporting the marketing authorization of biosimilars in Europe. Eur J Clin Pharmacol. 2020:76(4):557-66.

14. US Food and Drug Administration. Considerations in demonstrating interchangeability with a reference product: quidance for industry. In: U.S. Department of Health and Human Services, editor. Silver Spring: US Food and Drug Administration; 2019.

15. Kim H, Alten R, Avedano L, Dignass A, Gomollón F, Greveson K, et al. The future of biosimilars: maximizing benefits across immune-mediated inflammatory diseases. Drugs. 2020;80(2):99-113.

16. Leonard E, Wascovich M, Oskouei S, Gurz P, Carpenter D. Factors affecting health care provider knowledge and acceptance of biosimilar medicines: a systematic review. J Manag Care Spec Pharm. 2019:25(1):102-12

17. Sarnola K, Merikoski M, Jyrkkä J, Hämeen-Anttila K. Physicians' perceptions of the uptake of biosimilars: a systematic review. BMJ Open. 2020;10(5):e034183.
18. Gasteiger C, Lobo M, Dalbeth N, Petrie KJ. Patients' beliefs and behaviours are associated with perceptions of safety and concerns in a hypothetical biosimilar switch. Rheumatol Int. 2020:41(1):163-71.

19. Castañeda-Hernández G, Sandoval H, Coindreau J, Rodriguez-Davison LF, Pineda C. Barriers towards effective pharmacovigilance systems of biosimilars in rheumatology: a Latin American survey. Pharmacoepidemiol Drug Saf. 2019;28(8):1035-44.

20. O'Callaghan J, Barry SP, Bermingham M, Morris JM, Griffin BT. Regulation of biosimilar medicines and current perspectives on interchangeability and policy. Eur J Clin Pharmacol. 2019;75(1):1-11.

21. Moher D, Shamseer L, Clarke M, Ghersi D, Liberati A, Petticrew M, et al. Preferred reporting items for systematic review and meta-analysis protocols (PRISMA-P) 2015 statement. Syst Rev. 2015:4(1):1.

22. Moher D, Liberati A, Tetzlaff J, Altman DG, Group TP. Preferred reporting items for systematic reviews and meta-analyses: the PRISMA statement. PLoS Med. 2009;6(7):e1000097.

23. Higgins JP, Thomas J, Chandler J, Cumpston M, Li T, Page MJ, Welch VA, editors. Cochrane handbook for systematic reviews of interventions. Chichester (UK): Wiley; 2019

24. Treadwell JR, Uhl S, Tipton K, Shamliyan T, Viswanathan M, Berkman ND, et al. Assessing equivalence and noninferiority. J Clin Epidemiol 2012;65(11):1144-9.

25. Silvagni E, Bortoluzzi A, Carrara G, Zanetti A, Govoni M, Scirè CA. Comparative effectiveness of first-line biological monotherapy use in rheumatoid arthritis: a retrospective analysis of the RECord-linkage On Rheumatic Diseases study on health care administrative databases. BMJ Open. 2018;8(9):e021447.

26. Rathore AS, Bhargava A. Biosimilars in developed economies: overview, status, and regulatory considerations. Regul Toxicol Pharmacol. 2020;110:104525.

27. Declerck P, Danesi R, Petersel D, Jacobs I. The language of biosimilars: clarification, definitions, and regulatory aspects. Drugs. 2017;77(6):671-7.

28. Moots R, Azevedo V, Coindreau JL, Dörner T, Mahgoub E, Mysler E, et al. Switching between reference biologics and biosimilars for the treatment of rheumatology, gastroenterology, and dermatology inflammatory conditions: considerations for the clinician. Curr Rheumatol Rep. 2017;19(6):37.

29. Faccin F, Tebbey P, Alexander E, Wang X, Cui L, Albuquerque T. The design of clinical trials to support the switching and alternation of biosimilars. Expert Opin Biol Ther. 2016;16(12):1445-53.

30. Fleischmann R, Kremer J, Cush J, Schulze-Koops H, Connell CA, Bradley JD et al. Placebo-controlled trial of tofacitinib monotherapy in rheumatoid arthritis. N Engl J Med. 2012;367:495-507.

31. Keystone EC, Genovese MC, Klareskog L, Hsia EC, Hall ST, Miranda PC, et al. Golimumab, a human antibody to tumour necrosis factor a given by monthly subcutaneous injections, in active rheumatoid arthritis despite methotrexate therapy: the GO-FORWARD study. Ann Rheum Dis. 2009;68(6):789-96.

32. Felson DT, Anderson JJ, Boers M, Bombardier C, Furst D, Goldsmith C, et al American College of Rheumatology. Preliminary definition of improvement in rheumatoid arthritis. Arthritis Rheum. 1995;38:727-35.

33. Bruce B, Fries JF. The Stanford Health Assessment Questionnaire: a review of its history, issues, progress, and documentation. J Rheumatol. 2003;30(1):167-78.

34. Weir CJ, Butcher I, Assi V, Lewis SC, Murray GD, Langhorne P, et al. Dealing with missing standard deviation and mean values in meta-analysis of continuous outcomes: a systematic review. BMC Med Res Method. 2018;18(1):25.

35. da Costa BR, Nüesch E, Rutjes AW, Johnston BC, Reichenbach S, Trelle S, et al. Combining follow-up and change data is valid in meta-analyses of continuous outcomes: a meta-epidemiological study. J Clin Epidemiol. 2013;66(8):847-55

36. Higgins JPT, Green S, editors. Cochrane handbook for systematic reviews of interventions version 5.1.0. The Cochrane Collaboration; 2011. Available from: https://training.cochrane.org/handbook/archive/v5.1/.

37. Takeshima N, Sozu T, Tajika A, Ogawa Y, Hayasaka Y, Furukawa TA. Which is more generalizable, powerful and interpretable in meta-analyses, mean difference or standardized mean difference? BMC Med Res Methodol. 2014;14(1):30.

38. White IR. Multivariate random-effects meta-analysis. Stata J. 2009:9(1):40-56. 
39. Pereira TV, Patsopoulos NA, Salanti G, loannidis JP. Critical interpretation of Cochran's Q test depends on power and prior assumptions about heterogeneity. Res Synth Methods. 2010;1(2):149-61.

40. Borenstein M, Higgins JP, Hedges LV, Rothstein HR. Basics of meta-analysis: 12 is not an absolute measure of heterogeneity. Res Synth Methods. 2017;8(1):5-18.

41. Sterne JA, Sutton AJ, loannidis JP, Terrin N, Jones DR, Lau J, et al. Recommendations for examining and interpreting funnel plot asymmetry in meta-analyses of randomised controlled trials. BMJ. 2011;343:d4002

42. US Food and Drug Administration. Non-inferiority clinical trials to establish effectiveness: guidance for industry. In: U.S. Department of Health and Human Services, editor. Silverspring: US Food and Drug Adminstration; 2016.

43. Guyot P, Taylor PC, Christensen R, Pericleous L, Drost P, Eijgelshoven I, et al. Indirect treatment comparison of abatacept with methotrexate versus other biologic agents for active rheumatoid arthritis despite methotrexate therapy in the United Kingdom. J Rheumatol. 2012;39(6):1198-206.

44. Alonso-Ruiz A, Pijoan JI, Ansuategui E, Urkaregi A, Calabozo M, Quintana A. Tumor necrosis factor alpha drugs in rheumatoid arthritis: systematic review and metaanalysis of efficacy and safety. BMC Musculoskelet Disord. 2008;9:1.

45. Pope JE, Khanna D, Norrie D, Ouimet JM. The minimally important difference for the health assessment questionnaire in rheumatoid arthritis clinical practice is smaller than in randomized controlled trials. J Rheumatol. 2009;36(2):254-9.

46. Maini R, St Clair EW, Breedveld F, Furst D, Kalden J, Weisman M, et al. Infliximab (chimeric anti-tumour necrosis factor a monoclonal antibody) versus placebo in rheumatoid arthritis patients receiving concomitant methotrexate: a randomised phase III trial. Lancet. 1999;354(9194):1932-9.

47. Klareskog L, van der Heijde D, de Jager JP, Gough A, Kalden J, Malaise $M$, et al. Therapeutic effect of the combination of etanercept and methotrexate compared with each treatment alone in patients with rheumatoid arthritis: double-blind randomised controlled trial. Lancet. 2004;363(9410):675-81.

48. Strand V, Rentz AM, Cifaldi MA, Chen N, Roy S, Revicki D. Health-related quality of life outcomes of adalimumab for patients with early rheumatoid arthritis: results from a randomized multicenter study. J Rheumatol. 2012;39(1):63-72.
49. Schünemann H, Brożek J, Guyatt G, Oxman A, editors. GRADE handbook for grading quality of evidence and strength of recommendations. The GRADE Working Group; 2013. Updated October 2013. Available from: https://gdt.gradepro.org/app/handbook/handbook.html\#h.1i2bwkm8zp jo.

50. US Food and Drug Administration. FDA-approved drugs 2020. Available from: https://www.accessdata.fda.gov/scripts/cder/daf/.

51. European Medicines Agency. Medicines. EMA; 2020. Available from: https://www.ema.europa.eu/en/medicines.

52. Health Canada. Drug Product Database online query. Health Canada; 2020. Available from: https://health-products.canada.ca/dpd-bdpp/ index-eng.jsp.

53. Agência Nacional de Vigilância Sanitária. Consultas Medicamentos. ANVISA; 2020. Available from: https://consultas.anvisa.gov.br/\#/medic amentos/.

54. Moots RJ, Curiale C, Petersel D, Rolland C, Jones H, Mysler E. Efficacy and safety outcomes for originator TNF inhibitors and biosimilars in rheumatoid arthritis and psoriasis trials: a systematic literature review. BioDrugs. 2018;32(3):193-9.

55. Barbier L, Ebbers HC, Declerck P, Simoens S, Vulto AG, Huys I. The efficacy, safety, and immunogenicity of switching between reference biopharmaceuticals and biosimilars: a systematic review. Clin Pharmacol Therap. 2020;108(4):734-55

56. Huizinga TW, Torii Y, Muniz R. Adalimumab biosimilars in the treatment of rheumatoid arthritis: a systematic review of the evidence for biosimilarity. Rheumatol Ther. 2020;8:41-61.

57. Tanaka E, Kawahito Y, Kohno M, Hirata S, Kishimoto M, Kaneko Y, Tamai $H$, Seto Y, Morinobu A, Sugihara T, Murashima A. Systematic review and meta-analysis of biosimilar for the treatment of rheumatoid arthritis informing the 2020 update of the Japan College of Rheumatology clinical practice guidelines for the management of rheumatoid arthritis. Mod Rheumatol. 2021:6:1-13. https://doi.org/10.1080/14397595.2021.18995 91.

\section{Publisher's Note}

Springer Nature remains neutral with regard to jurisdictional claims in published maps and institutional affiliations.
Ready to submit your research? Choose BMC and benefit from:

- fast, convenient online submission

- thorough peer review by experienced researchers in your field

- rapid publication on acceptance

- support for research data, including large and complex data types

- gold Open Access which fosters wider collaboration and increased citations

- maximum visibility for your research: over $100 \mathrm{M}$ website views per year

At BMC, research is always in progress.

Learn more biomedcentral.com/submissions 